\title{
Factors Affecting the Relative Intensity Noise of GaN Quantum Dot Lasers
}

\author{
Hussein B. AL-Husseini \\ Nassiriya Nanotechnology Research Laboratory (NNRL) \\ Physics Department, Science College, Thi-Qar University, Nassiriya
}

Iraq

\section{Introduction}

The motivation for writing this chapter is at least threefold: the extremely promising laser characteristics of GaN quantum dot semiconductor lasers; their vast application potential in high-power and high-temperature optoelectronic devices like optical communications, industrial manufacturing, medicine, military uses, sensing and optical coherent tomography. Low noise is one of the requirements in the wide range of applications that one hopes to arrive at in the use of optoelectronic devices that deals with QD structures. When the size of an electron system reaches the nanometer scale, noise becomes a very interesting problem [2]. Its origin lies in spontaneous emission and shot noise; the last of which is a nonequilibrium fluctuation, caused by the discreteness of the charge carriers. From the investigation of shot noise, one can learn additional information on electronic structure and transport properties. It is directly related to the degree of randomness in carrier transfer $[2,3]$. In most experimental studies, it is assumed [4] that the frequency fluctuation processes are either infinitely fast, leading to homogeneous broadening, or infinitely slow, leading to inhomogeneous broadening. However, effects of a spectral fluctuation on a picosecond time scale have been observed in experiments, showing that the frequency fluctuation [4] is not always infinitely fast or slow. The random forces have no memory and the correlation of their product function is a delta function. The memory effects [6,7] arise because the wave functions of the particles are smeared out so that there is always some overlap of wave functions. As a result, the particle retains some memory of the collisions experienced through its correlation with other particles in the system.

Quantum dot semiconductor lasers is the subject of intense investigations for the last and this decades owing to their unique properties that arise from 3-D quantum confinement. This is due to the expected characteristics like: Low threshold current density, high characteristic temperature and small linewidth enhancement factor (LEF) [1]. This latter property should lead to an increased tolerance to optical noise with the perspective of achieving isolator-free lasers, of particular importance for low-cost lasers in metropolitan and local area networks [8]. The peculiar property of the semiconductor QDs grown by selfassembling are: 1) the strong compressive strain inside the QDs which leads to a significant deformation of the potentials respect to the bulk case [9], 2) the inhomogeneous distribution of the QD size [10], [11] and 3) the presence of more than one confined energy state inside 
the QDs [12]. The origin of laser fluctuations in intensity and phase lies in the quantum nature of lasing process itself. These fluctuations are of great importance since they introduce errors in optical communications. In fact, every spontaneous emission event in the oscillating mode, varying the phase of the electromagnetic field (quantum noise) is responsible for the carrier density variation [13].

In this chapter, we report on the RIN performance of $\mathrm{GaN} / \mathrm{Al}_{0.25} \mathrm{Ga}_{0.75} \mathrm{~N} / \mathrm{AlN}$ QD laser. The influence of power and relaxation time, as well as the intensity noise properties of the input signals, is investigated. It will be shown that improvement in the RIN performance is possible under certain operating conditions. This paper is organized as follows. In Section II, the general aspects of our investigation are presented, as well as the derivation of the RIN in QDs. The results are presented and discussed in Section III. Finally, the current and future developments are given in Section IV.

In the recent years we have seen an ever increasing number of papers and conferences dealing with theory and experiments with QD devices. This has produced a huge amount of information and sometimes contradictory results. As a consequence it can be difficult for new starters finding the right way for getting a basic understanding of the physics of QD devices. In this chapter we have therefore introduced several references to those papers we judged to be fundamental for understanding the basic physics of QD lasers with the hope of providing a useful guideline for those who start adventuring in this field.

\section{Relative intensity noise}

Relative intensity noise (RIN) is studied by introducing the Langevin noise terms in the laser rate equations (REs) as a driving force where the REs of the carrier-number, or -density, for the active, barrier and SCH layers are used [14, 15]. Here, we modeled the REs by using occupation probability of carriers in the QD, WL and SCH layers. The QDs used here are assumed to be in the form of a disk shape. The laser structure studied is illustrated in Fig.(1). The energy diagram of the QD laser active region is depicted in Fig. (2). QD states are taken into account here by introducing the excited state (ES) in addition to the ground state (GS). The WL, which is in the form of a quantum well, is represented by one energy state [16]. The Pauli-blocking due to state filling is taken into account. The resulting REs system including noise then becomes,

$$
\begin{gathered}
\frac{d f_{s c h}}{d t}=-\frac{f_{s c h}\left(1-f_{w l}\right)}{\tau_{c o, s c h}}+\frac{f_{w l}}{\tau_{e, w l}}-\frac{f_{s c h}}{\tau_{s r}}+F_{f_{s c h}}(t) \\
\frac{d f_{w l}}{d t}=\left(\frac{f_{s c h}}{\tau_{c o, s c h}}+\frac{4 f_{E S}}{\tau_{e o, E S}}\right)\left(1-f_{w l}\right)-\frac{f_{w l}}{\tau_{e, w l}}-\frac{f_{w l}}{\tau_{c o, w l}}\left(1-f_{E S}\right)-\frac{f_{w l}}{\tau_{w r}} \\
+F_{f_{w l}}(t) \\
\frac{d f_{E S}}{d t}=\left(\frac{f_{w l}}{4 \tau_{c o, w l}}+\frac{f_{G S}}{2 \tau_{e o, G S}}\right)\left(1-f_{E S}\right)-\frac{f_{E S}}{\tau_{e o, E S}}\left(1-f_{w l}\right)-\frac{f_{E S}}{\tau_{c o, E S}}\left(1-f_{G S}\right) \\
-\frac{f_{E S}}{\tau_{r}}+F_{f_{E S}}(t)
\end{gathered}
$$




$$
\begin{gathered}
\frac{d f_{G S}}{d t}=\frac{2 f_{E S}\left(1-f_{G S}\right)}{\tau_{c o, E S}}-\frac{f_{G S}\left(1-f_{E S}\right)}{\tau_{e o, G S}}-\frac{f_{G S}}{\tau_{r}}-v_{g} \Gamma g_{G S} S_{p}+F_{f_{G S}}(t) \\
\frac{d S_{p}}{d t}=v_{g} \Gamma g_{G S} S_{p}-\frac{S_{p}}{\tau_{p}}+\beta \frac{2 f_{G S}}{\tau_{r}}+F_{f_{S}}(t)
\end{gathered}
$$

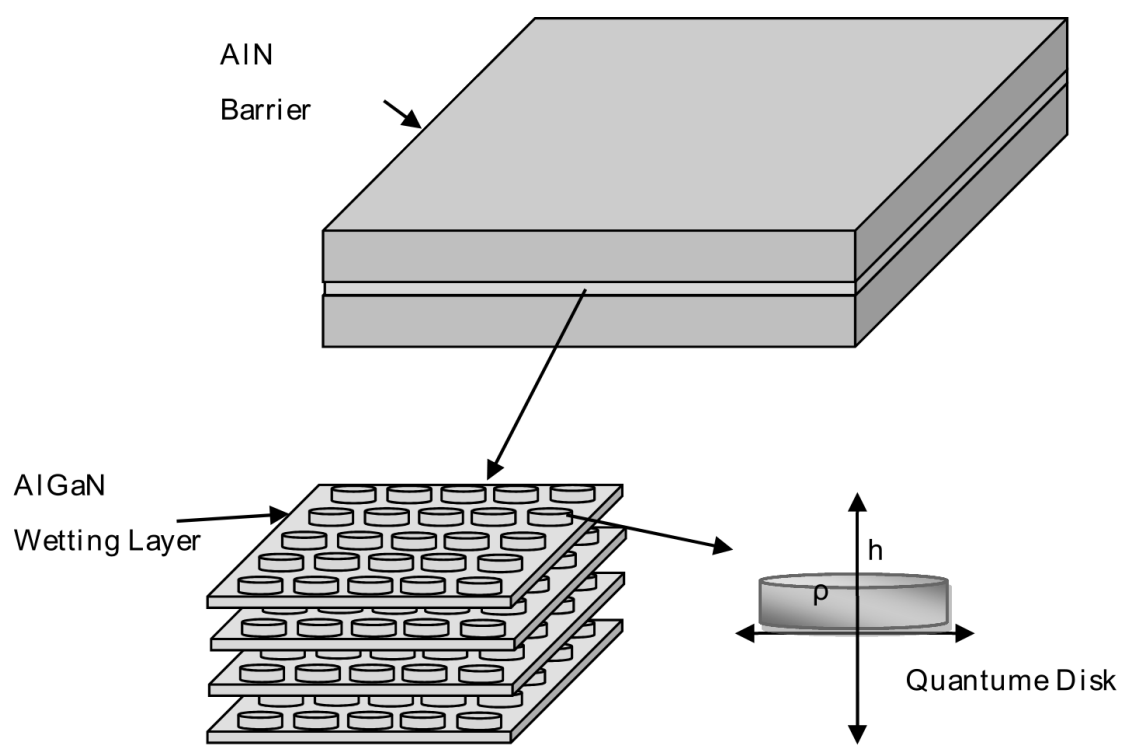

Quantume Dots Multi Layers

Fig. 1. Schematic illustration of the QD active layer which consists of an arrays of GaN quantum disks grown on an AlGaN wetting layer covered by AlN barrier.

The occupation probabilities for the GS and ES are defined as $f_{G S}=n_{G S} / 2 N_{D}$ and $f_{E S}=n_{E S} / 4 N_{D}$, where $n_{G S}\left(n_{E S}\right)$ is the number of electron-hole $(e-h)$ pairs in the GS $(E S), N_{D}$ is the total number of QDs, $g_{G S}$ is the GS gain while the SCH and WL populations and the photon occupation are described by the normalized terms: $f_{s c h}=n_{s c h} N_{D}, f_{w l}=n_{w l} N_{D}$, and $S_{p}=n_{p} N_{D}$, respectively. $n_{p}$ is the photon number, $\Gamma$ is the optical confinement factor. In Eq. (5), the last term on the righthand side $\left(2 \beta f_{G S} / \tau_{r}\right)$ represents the rate of photons emitted by spontaneous emission coupled into the lasing mode. Note that, the size inhomogeneity of the dots is included in the expression of the gain. The capture and escape times; in Eqs. (1-4) are given by [1]

$$
\begin{aligned}
& \tau_{c, s c h}^{-1}=\left(1-f_{w l}\right) \tau_{c o, s c h}^{-1} \\
& \tau_{c, w l}^{-1}=\left(1-f_{E S}\right) \tau_{c o, w l}^{-1} \\
& \tau_{c . E S}^{-1}=\left(1-f_{G S}\right) \tau_{c o, E S}^{-1} \\
& \tau_{e, G S}^{-1}=\left(1-f_{E S}\right) \tau_{e o, G S}^{-1} \\
& \tau_{e, E S}^{-1}=\left(1-f_{w l}\right) \tau_{e o, E S}^{-1}
\end{aligned}
$$




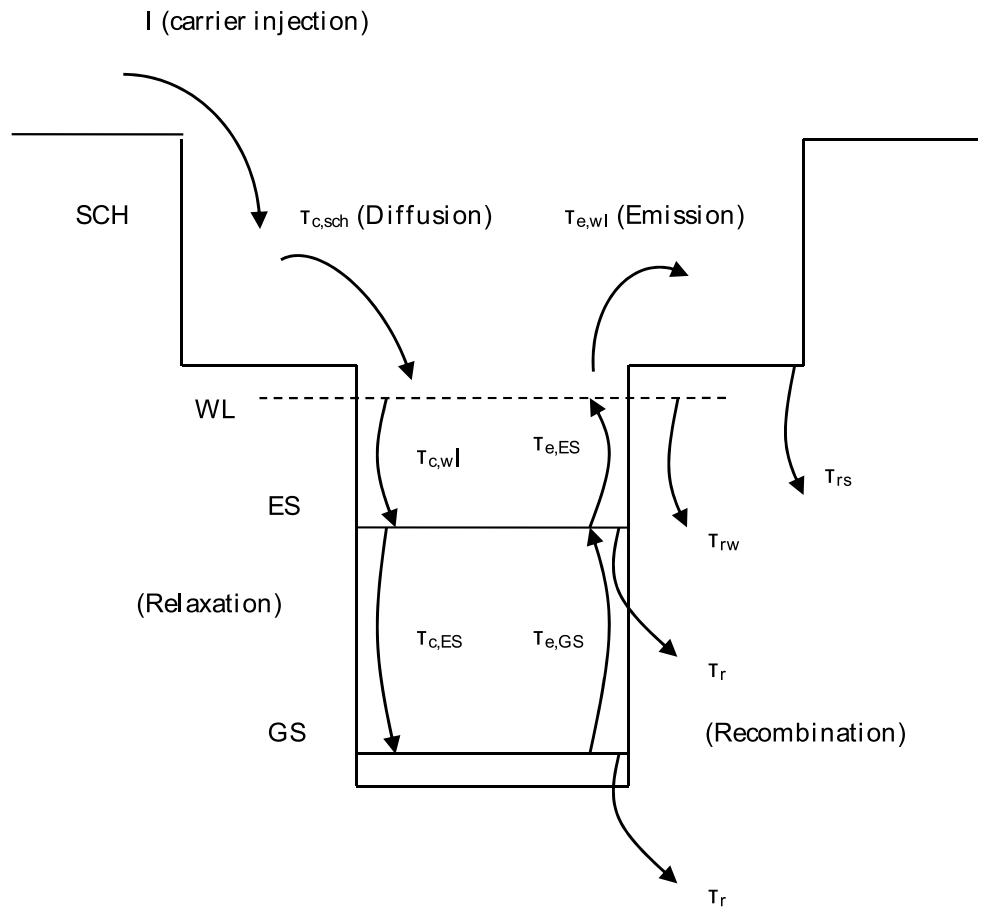

Fig. 2. Energy diagram of the active layer of the QD laser.

$\tau_{c, s c h}, \tau_{c, w l}$, and $\tau_{c, E S}$ are the average capture times from $\mathrm{SCH}$ to $\mathrm{WL}, \mathrm{WL}$ to ES and from QD ES to GS, respectively, with the hypothesis that the final state is empty. The emission times $\tau_{e, G S}$, and $\tau_{e, E S}$ are the escape times from the GS back to the ES and from the ES back to the WL. $\tau_{0-1}$ is the relaxation rate when the state is unoccupied, i.e., $f=0$. As $f$ approaches 1 the relaxation rate decreases, resulting in the occupation of the upper level. Furthermore, at room temperature and before reaching stimulated emission the system must converge to a quasi-thermal equilibrium characterized by a Fermi distribution of the carriers in all the states. The carrier transport time from the SCH to WL or QD can be expressed as [13],

$$
\tau_{c}=\frac{d_{s}^{2}}{2 D_{\text {n.p }}}
$$

Where $d_{s}$ is the thickness of the SCH layer, $D_{n}$ and $D_{p}$ are the diffusion coefficients of electrons and holes, respectively, which can be calculated from the Einstein relation $D_{n, p}=\left(K_{B} T / q\right) \mu_{n, p}$, where $K_{B}, T, \mu_{n, p}$, correspond to the Boltzmann's constant, lattice temperature, electron and hole mobility, respectively. The thermionic emission time in the QD can be calculated by [15]

$$
\tau_{e}=d_{w}\left[\frac{2 \pi m_{e}^{*}}{K_{B} T}\right]^{1 / 2} \exp \left(\frac{E_{b}}{K_{B} T}\right)
$$


where $d_{w}, m_{e}^{*}, E_{b}$ denote the thickness of the QD, electron effective mass and the effective barrier energy, respectively. The carrier relaxation time $\tau_{r}$ in general, is about 2.8-4.5 ns for typical quantum well laser [15]. The thickness of the SCH (WL) is 33nm (5nm). In Eqs (1-5) $F_{f s c h}, F_{f w l}, F_{f E S}, F_{f G S}$ and $F_{f s}$ are the Langevin noise forces. Physically $F_{f s}$ arise from spontaneous emission, while $F_{f s c h}, F_{f w l}, F_{f E S}$ and $F_{f G S}$ have their origin in the discrete nature of the carrier generation and recombination processes in the $\mathrm{SCH}, \mathrm{WL}$, ES and GS region, respectively. The small-signal analysis [1] (along with the Fourier transform) is used to get RIN spectra for these systems. It is defined as $R I N=S_{s}(w) / S_{0}^{2}$ where $S_{s}(w)=\left\langle|\hat{s}(w)|^{2}\right\rangle$ is the spectral fluctuation of photon population around its steady-state value (intensity noise). The angle brackets denotes ensemble average. The small-signal solution of the REs (1-5) yields the following expression

$$
\begin{aligned}
\hat{s}(w)= & A_{f_{s c h}}(w) \cdot \hat{F}_{f_{s c h}}(w)+A_{f_{w l}}(w) \cdot \hat{F}_{f_{w l}}(w)+A_{f_{E S}}(w) \cdot \hat{F}_{f_{E S}}(w) \\
& +A_{f_{G S}}(w) \cdot \hat{F}_{f_{G S}}(w)+A_{s}(w) \cdot \hat{F}_{S}(w)
\end{aligned}
$$

where $\widehat{F}_{f}$ and $\widehat{F}_{s}$ are the Fourier transforms of the Langevin forces. We recall, however, that these Fourier transforms do not possess a mathematical existence, where

$$
\begin{gathered}
A_{f_{s c h}}(w)=\frac{a_{21} a_{32} a_{43} a_{54}}{D(w)} \\
A_{f_{w l}}(w)=\frac{a_{32} a_{43} a_{54}\left(a_{11}+j w\right)}{D(w)} \\
A_{f_{E S}}(w)=\frac{a_{43} a_{54}\left[-a_{12} a_{21}-\left(a_{11}+j w\right)\left(a_{22}-j w\right)\right]}{D(w)} \\
A_{f_{G S}}(w)=\frac{\left.\left(a_{11}+j w\right)\left(a_{22}-j w\right)\left(a_{33}-j w\right)\right]}{D(w)} \\
a_{s}[w)=\frac{-a_{23} a_{32}\left(a_{11}+j w\right)+a_{12} a_{21}\left(a_{33}-j w\right)+}{\left.-a_{34} a_{43}\left(a_{11}+j w\right)\left(a_{22}-j w\right)-\left(a_{11}+j w\right)\left(a_{22}-j w\right)\left(a_{33}-j w\right)\left(a_{44}-j w\right)\right]} \\
D(w)
\end{gathered}
$$

The denominator $\mathrm{D}(\mathrm{w})$ is given by

$$
D(w)=A_{o}+A_{1}(j w)+A_{2}(j w)^{2}+A_{3}(j w)^{3}+A_{4}(j w)^{4}+(j w)^{5}
$$

Here $A_{1}, A_{2}, A_{3}, A_{4}$ are defined in Appendices $\mathrm{A}$ and $\mathrm{B}$. It is clear that the RIN is due to the contributions of the noise sources $F_{f s c h}, F_{f w l}, F_{f E S}, F_{f G S}$ and $F_{f s}$. The Langevin forces satisfy the general relations $[16,17]$

$$
\left\langle F_{i}(t)\right\rangle=0
$$




$$
\left\langle F_{i}(t) F_{j}\left(t^{\prime}\right)\right\rangle=2 D_{i j} \delta\left(t-t^{\prime}\right)
$$

where $D_{i j}$ is the diffusion coefficient associated with the corresponding noise source. According to our analysis, we found the following nonzero steady-state diffusion coefficients $D_{i j}$ given by

$$
\begin{aligned}
& D_{f_{s c h} f_{s c h}}=\frac{f_{s c h}}{\tau_{11}\left(f_{s c h}\right)} \\
& D_{f_{w l} f_{w l}}=\frac{f_{w l}}{\tau_{22}\left(f_{w l}\right)} \\
& D_{f_{E S} f_{E S}}=\frac{f_{E S}}{\tau_{33}\left(f_{E S}\right)} \\
& D_{f_{G S} f_{G S}}=v_{g} \Gamma g_{G S} S_{p} \\
& D_{S_{p} f_{G S}}=-D_{f_{G S} f_{G S}} \\
& D_{S_{p} S_{p}}=\beta \frac{2 f_{G S}}{\tau_{r}} S_{o}
\end{aligned}
$$

Where $1 / \tau_{i j}$ is the carrier lifetime in the $\mathrm{SCH}, \mathrm{WL}$ and QD regions [1]. Using Eqs. (11) and (12), the spectral density of the intensity noise is given by the following expression

$$
\begin{aligned}
S_{s}(w)= & 2\left|A_{f_{s c h}}(w)\right|^{2} \frac{f_{s c h}}{\tau_{11}\left(f_{s c h}\right)}+2\left|A_{f_{w l}}(w)\right|^{2} \frac{f_{w l}}{\tau_{22}\left(f_{w l}\right)}+ \\
& 2\left|A_{f_{E S}}(w)\right|^{2} \frac{f_{E S}}{\tau_{33}\left(f_{E S}\right)}+2\left|A_{f_{G S}}(w)\right|^{2} v_{g} \Gamma g_{G S} S_{p}+ \\
& 2\left|A_{s}(w)\right|^{2} \beta \frac{2 f_{G S}}{\tau_{r}} S_{o}+4\left[\operatorname{Re}\left(A_{f_{G S}}(w)\right) \cdot \operatorname{Re}\left(A_{s}(w)\right)\right. \\
& \left.+\operatorname{Im}\left(A_{f_{G S}}(w)\right) \cdot \operatorname{Im}\left(A_{s}(w)\right)\right]\left(-v_{g} \Gamma g_{G S} S_{p}\right)
\end{aligned}
$$

Where Re (.) and IM (.) stands respectively, for the real and imaginary parts of the complex argument.

To see the origin of this RIN model let us compare it with the earlier rate equation models. Properties including structure dependence, in this model, are to be discussed elsewhere. Its effect on the modulation response in the carrier transport model is discussed in Ref. [11]. One can see that

1. Setting is $\tau_{c o, w l}=0, \tau_{e, w l}=\infty, \tau_{w r}=\infty$ equivalent to neglecting the effect of the WL. This returns it to the three-level carrier transport model as in Ref. [12].

2. After making the assumption described for (1), this returns it to the earlier one where it becomes only the active region (QD-region here) which is taken in the analysis as in Ref. [8].

Accordingly, the RIN can be split into six components by make each equation refers to only force effecting without the correlation with other forces in the system. 


$$
\begin{aligned}
& \text { (1) }\left.R I N\right|_{F_{\text {sch }} . F_{\text {sch }}}=2\left|A_{f_{\text {sch }}}(w)\right|^{2}\left\langle F_{\text {sch }} \cdot F_{\text {sch }}\right\rangle \\
& \text { (2) }\left.R I N\right|_{F_{w l} \cdot F_{w l}}=2\left|A_{f_{w l}}(w)\right|^{2}\left\langle F_{w l} \cdot F_{w l}\right\rangle \\
& \text { (3) }\left.R I N\right|_{F_{E S} . F_{E S}}=2\left|A_{f_{E S}}(w)\right|^{2}\left\langle F_{E S} \cdot F_{E S}\right\rangle \\
& \text { (4) RIN }\left.\right|_{F_{G S} \cdot F_{G S}}=2\left|A_{f_{G S}}(w)\right|^{2}\left\langle F_{G S} \cdot F_{G S}\right\rangle \\
& \text { (5) }\left.R I N\right|_{F_{s p}, F_{s p}}=2\left|A_{s}(w)\right|^{2}\left\langle F_{s p} . F_{s p}\right\rangle \\
& \text { (6) }\left.R I N\right|_{F_{G S} \cdot F_{s p}}=4\left[\operatorname{Re}\left(A_{f_{G S}}(w)\right) \cdot \operatorname{Re}\left(A_{s}(w)\right)\right. \\
& \left.+\operatorname{Im}\left(A_{f_{G S}}(w)\right) \cdot \operatorname{Im}\left(A_{s}(w)\right)\right]\left\langle F_{G S} \cdot F_{s p}\right\rangle
\end{aligned}
$$

$\left.R I N\right|_{F_{G S} . F_{s p}}$ denotes that the RIN is due to the mixing of the two noise sources, $F_{G S}$ and $F_{S P}$.

\section{Results and discussion}

The GaN/ $\mathrm{Al}_{0.25} \mathrm{Ga}_{0.75} \mathrm{~N} / \mathrm{AlN}$ QD laser structure is taken as an example for relative intensity noise study. GaN dots studied here are assumed to have a disk shape with a radius of (14 $\mathrm{nm})$ and $(2 \mathrm{~nm})$ height. Its maximum gain $\left(1800 \mathrm{~cm}^{-1}\right)$ appears at $(\lambda=345 \mathrm{~nm}(3.6 \mathrm{eV}))$. The differential linear gain and the transparency surface carrier density (the carrier density at zero gain) values are calculated, respectively, as $\left(3.35 \times 10^{18} \mathrm{~cm}^{2}\right.$ and $\left.1.54 \times 10^{12} \mathrm{~cm}^{-2}\right)$. The RIN for $\mathrm{GaN} / \mathrm{Al}_{0.25} \mathrm{Ga}_{0.75} \mathrm{~N} / \mathrm{AlN}$ QD lasers has been computed using Eq. (13). The parameters used in the calculation are listed in Table 1. Fig. 3 shows contributions of RIN components results due to effect of: $\mathrm{SCH}\left(F_{S C H}\right), \mathrm{WL}\left(F_{W L}\right), \mathrm{ES}\left(F_{E S}\right), \mathrm{GS}\left(F_{G S}\right)$ and that part results from photon density $\left(F_{S P}\right)$. These components are listed in Eq. (14). It is shown that the main noise contribution comes from WL and ES. This also with our conclusion about the chaotic behavior of QD structures [18]. RIN pronounced peak from relaxation oscillations at resonance frequency of $5 \mathrm{GHz}$, Fig. 4 shows ES contribution to noise $\left(F_{E S}\right)$ at two ES recombination times compared with RIN spectrum. Longer recombination time reduces the noise. Fig. 5 shows the recombination time effect on the WL contribution to noise $\left(F_{W L}\right)$. It is shown that this time has more pronounced effect on reducing WL contribution. Noise reduction due to recombination can be attributed to reduction of threshold current which can reduce noise. Both figures (4and 5) show a different situation when the recombination time ( $\left.\tau_{33}\right)$ in the QD region becomes the variable parameter. The larger effect of $\tau_{33}$ on RIN spectra is obvious. At ( $\left.\tau_{33} 2.8 \mathrm{ps}\right)$ the relaxation oscillation frequency begins to retard.

\begin{tabular}{|c||}
\hline Laser parameters \\
\hline \hline Active region length, $\mathrm{L}=900 \mathrm{~nm}$ \\
\hline Active region width, $\mathrm{W}=0.1 \mu \mathrm{m}$ \\
\hline Number of QD layers, $\mathrm{N}_{\mathrm{w}}=10$ \\
\hline QD density per unit area, $\mathrm{N}_{\mathrm{d}}=5 \times 10^{12} \mathrm{~cm}^{-2}$ \\
\hline Internal loss, $\mathrm{a}_{\text {int }}=3 \mathrm{~cm}^{-1}$ \\
\hline Spontaneous emission factor, $\beta=10^{-4}$ \\
\hline \hline
\end{tabular}

Table 1. Parameters of the QD material and laser [1] 


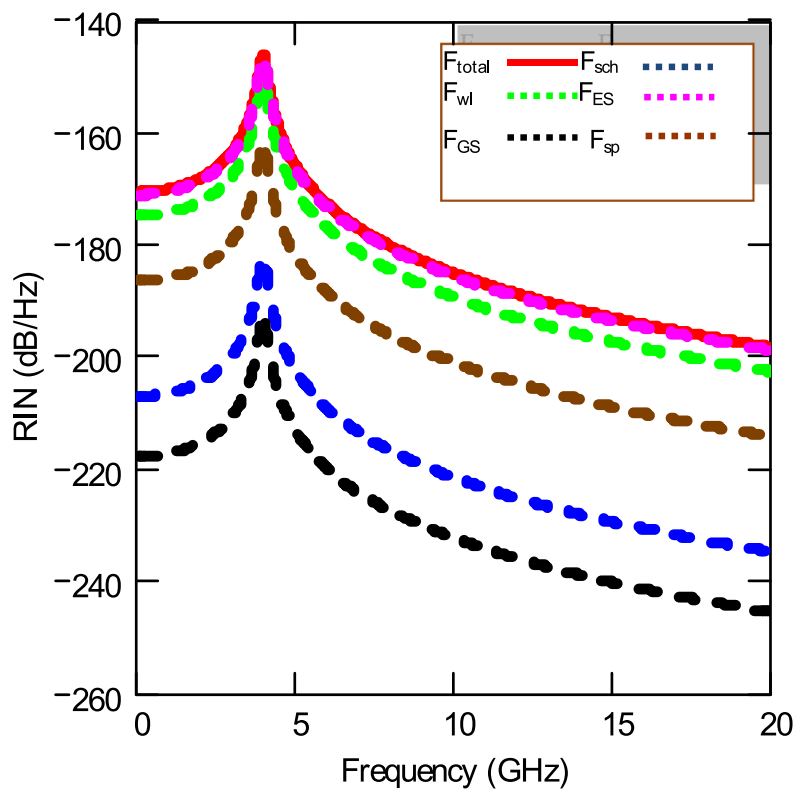

Fig. 3. The RIN of $\mathrm{GaN} / \mathrm{Al}_{0.25} \mathrm{Ga}_{0.75} \mathrm{~N} / \mathrm{AlN}$ QD lasers with different contributions of RIN parts: $S C H$ part $\left(F_{S C H}\right)$, WL part $\left(F_{W L}\right)$, ES part $\left(F_{E S}\right), G S$ part $\left(F_{G S}\right)$. The RIN spectrum for the structure is referred to by $F_{\text {total }}$ in the inset.

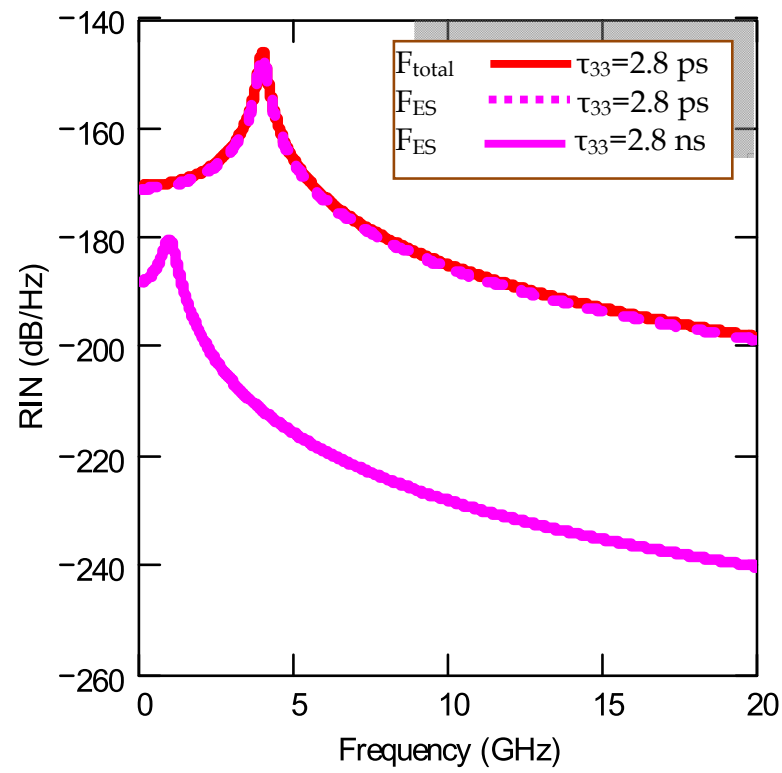

Fig. 4. The RIN of $\mathrm{GaN} / \mathrm{Al}_{0.25} \mathrm{Ga}_{0.75} \mathrm{~N} / \mathrm{AlN}$ QD lasers compared with the contribution of RIN from ES $\left(F_{E S}\right)$ at two QD recombination times. The RIN spectrum for the structure is referred to in the inset as $F_{\text {total }}$. 


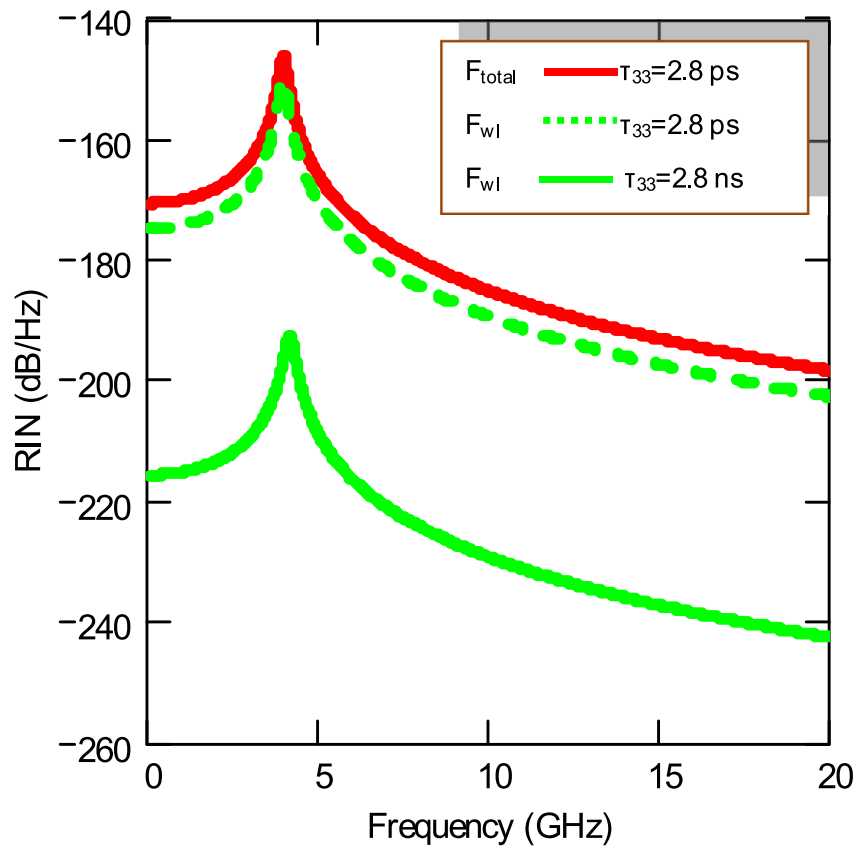

Fig. 5. The RIN of $\mathrm{GaN} / \mathrm{Al}_{0.25} \mathrm{Ga}_{0.75} \mathrm{~N} / \mathrm{AIN}$ QD lasers compared with the contribution of RIN from WL $\left(F_{W L}\right)$ at two QD recombination times. The RIN spectrum for the structure is referred to in the inset as $F_{\text {total }}$.

\section{Appendix A}

All the partial derivatives coefficients $a_{\mathrm{ij}}$ are real quantities evaluated at steady-state values $f_{\text {scho }}, f_{\text {wlo }}, f_{E S o}, f_{G S o}$ and $S_{\text {pos. }}$.

$$
\begin{gathered}
a_{11}=\frac{1}{\tau_{11}\left(f_{\text {sch }}\right)}+\frac{1}{\tau_{12}}\left(1-f_{w l}\right), a_{12}=-\frac{1}{\tau_{21}}, a_{23}=\frac{4}{\tau_{32}}\left(1-f_{w l}\right) \\
a_{21}=\frac{\left(1-f_{w l}\right)}{\tau_{12}}, a_{22}=-\frac{1}{\tau_{22}\left(f_{w l}\right)}-\frac{1}{\tau_{21}}-\frac{1}{\tau_{23}}\left(1-f_{E S}\right), a_{32}=\frac{1}{4 \tau_{23}}\left(1-f_{E S}\right) \\
a_{34}=\frac{\left(1-f_{E S}\right)}{2 \tau_{43}}, a_{33}=-\frac{1}{\tau_{33}\left(f_{E S}\right)}-\frac{1}{\tau_{32}}\left(1-f_{w l}\right)-\frac{1}{\tau_{34}}\left(1-f_{G S}\right)
\end{gathered}
$$




$$
\begin{aligned}
& a_{43}=\frac{2}{\tau_{34}}\left(1-f_{G S}\right), a_{44}=-\frac{1}{\tau_{44}\left(f_{G S}\right)}-\frac{1}{\tau_{43}}\left(1-f_{E S}\right)-G_{f_{G S}} S_{0} \\
& a_{45}=-G_{0}-G_{S} S_{0}, a_{55}=G_{0}+G_{S} S_{p o}-\frac{1}{\tau_{p}}, a_{54}=G_{f_{G S}} S_{p o}+\beta \frac{2}{\tau_{r}}
\end{aligned}
$$

\section{Appendix B}

$$
\begin{aligned}
A_{o}= & -a_{11} a_{23} a_{32} a_{44} a_{55}-a_{11} a_{22} a_{43} a_{34} a_{55}+a_{11} a_{23} a_{32} a_{45} a_{54}+a_{11} a_{22} a_{33} a_{44} a_{55} \\
& -a_{12} a_{21} a_{34} a_{43} a_{55}+a_{12} a_{21} a_{33} a_{44} a_{55}-a_{12} a_{21} a_{33} a_{45} a_{54}-a_{11} a_{22} a_{33} a_{45} a_{54} \\
A_{1}= & a_{11} a_{23} a_{32} a_{55}+a_{11} a_{22} a_{43} a_{34}-a_{11} a_{22} a_{33} a_{44}-a_{11} a_{22} a_{33} a_{55}+a_{11} a_{23} a_{32} a_{44} \\
& -a_{44} a_{11} a_{22} a_{55}+a_{11} a_{22} a_{45} a_{54}+a_{55} a_{34} a_{11} a_{43}-a_{55} a_{44} a_{33} a_{11}+a_{33} a_{11} a_{45} a_{54} \\
& +a_{55} a_{44} a_{23} a_{32}+a_{45} a_{23} a_{32} a_{54}-a_{55} a_{34} a_{22} a_{43}+a_{55} a_{44} a_{33} a_{22}-a_{33} a_{22} a_{45} a_{54} \\
& +a_{12} a_{21} a_{34} a_{43}-a_{55} a_{12} a_{21} a_{33}-a_{12} a_{21} a_{33} a_{44}-a_{55} a_{44} a_{12} a_{21}+a_{12} a_{21} a_{45} a_{54} \\
A_{2}= & -a_{11} a_{23} a_{32}+a_{11} a_{22} a_{33}+a_{44} a_{11} a_{22}+a_{55} a_{11} a_{22}-a_{34} a_{11} a_{43}+a_{44} a_{33} a_{11} \\
& +a_{55} a_{33} a_{11}+a_{55} a_{11} a_{44}-a_{11} a_{45} a_{54}-a_{44} a_{23} a_{32}+a_{55} a_{23} a_{32}+a_{34} a_{22} a_{43} \\
& -a_{44} a_{33} a_{22}-a_{55} a_{33} a_{22}-a_{55} a_{44} a_{22}+a_{22} a_{45} a_{54}-a_{55} a_{44} a_{33}+a_{55} a_{34} a_{43} \\
& +a_{33} a_{45} a_{54}+a_{12} a_{21} a_{33}+a_{55} a_{12} a_{21}+a_{44} a_{12} a_{21} \\
A_{3}= & -a_{11} a_{22}-a_{33} a_{11}-a_{11} a_{44}-a_{55} a_{11}-a_{23} a_{32}+a_{33} a_{22}+a_{44} a_{22}+a_{55} a_{22} \\
& -a_{45} a_{54}-a_{34} a_{43}+a_{44} a_{33}+a_{55} a_{33}+a_{55} a_{44}-a_{12} a_{21} \\
& A_{4}=a_{11}-a_{22}-a_{33}-a_{44}-a_{55}
\end{aligned}
$$

\section{Conclusions}

Relative intensity noise characteristics in QD structure are studied using The resulting REs system including noise. This enables us to study relaxation, recombination and emission processes in the QD region. This is impossible with other models. GaN QDs are taken as an example for the study. The following points can be stated:

1. Most of the contributions due to carriers in both wetting layer and excited state of QD have a main contribution in noise. This gives the importance of carrier dynamics in the QD and WL regions .

2. $\tau_{33}$ governs the effects of emission from - and recombination outside QD region, then it can reduce the intensity noise at some short value.

From these results one can conclude the important effect of the phonon-bottleneck on intensity noise behavior of these structures. Crystal quality and smaller recombination and relaxation times inside the dot reduce the noise. 


\section{Acknowledgments}

The author express his deep gratitude for the support provided by the Proof. Dr. Amin H. AL-Khursan, head of (NNLR) for project control and guidance.

\section{References}

[1] H. Al-Husseini, Amin H. Al-Khursan, S. Y. Al -Dabagh, "III-Nitride QD lasers”, Open Nanosci J. 3, 1-11, 2009.

[2] H.K. Zhao, "Shot noise in the hybrid systems with a quantum dot coupled to normal and superconducting leads", Phys. Lett. A 299 (2002) 262.

[3] K.Y. Lau, in: P.S. Zory (Ed.), Quantum Well Lasers, Dynamics of Quantum Well Lasers, Academic Press, New York, 1993, p. 252 (Chapter 5).

[4] H.J. Bakker, K. Leo, J. Shah, K. Kohler, Phys. Rev. B 49 (1994) 8249.

[5] G.P. Agrawal, N.K. Dutta, Long Wavelength Semiconductor Lasers, Van Nostrand Reinhold, New York, 1986 (Chapter 9).

[6] D. Ahn, IEEE J. Select. Top. Quantum Electron. 1 (1995) 301.

[7] D. Ahn, Phys. Rev. B 51 (1995) 2159.

[8] T. Steiner, Semiconductor Nanostructures for Optoelectronic Applications. British Library Cataloguing in Publication Data London: Boston, 2004.

[9] M. Grundman, O. Stier, and D. Bimberg, "InGs/GaAs pyramidal quantum dots: Strain distribution, optical phonons, and electronic structure," Phys. Rev. B 52, vol. 52, no. 16, pp. 11969 - 11 981, October 1995.

[10] O. Qasaimeh, "Efffect of inhomogeneous line broadening on gain and differential gain of quantum dot lasers," IEEE Tran. Electron. Dev., vol. 50, no. 7, pp. 1575-1581, July 2003.

[11] M. Gioannini, "Numerical modelling of the emission characteristics of semiconductor quantum dash materials for lasers and optical amplifiers," IEEE J. Quantum Electron., vol. 40, no. 4, April 2004.

[12] A. Markus, J. Chen, O. G.-L. J. Provost, C. Paranthoen, and A. Fiore, "Impact of intraband relaxation on the performance of a quantum-dot laser," IEEE J. Select. Top. Quantum Electron., vol. 9, no. 5, pp. 1308-1314, September/October 2003.

[13] C. Z. Tong , S. F. Yoon, C. Y. Ngo, C. Y. Liu, W. K. Loke, "Rate equations for 1.3- $\mu \mathrm{m}$ dots-under-a-well and dots-in-a-well self-assembled InAs-GaAs quantum-dot lasers", IEEE J. Quantum Electron., 42, 1175-83, 2006.

[14] Amin H. Al-Khursan, "Intensity noise characteristics in quantum-dot lasers: Four-level rate equations analysis" J. Lumin, 113, 129-36, 2005.

[15] S. Schulz, G. Czycholl, "Spin-orbit coupling and crystal-field splitting in the electronic and optical properties of nitride quantum dots with a wurtzite crystal structure", Condens. Matter Mater. Sci., 1, 2436, 2008.

[16] U. Goesele, S. Senz, V. Schmidt, Y. Wang, C. Hangzhou, "Process for group III-V semiconductor nanostructure synthesis and compositions made using same" US7557028 (2009). 
[17] M. Gioannini, Amin H. Al-Khursan, G. A.P. Thé and I. Montrosset, "Simulation of quantum dot lasers with weak external optical feedback", Dynamics Days Conference, Delft-Netherlands, 2008.

[18] H. Al-Husseini, Amin H. Al-Khursan, "Relative Intensity Noise for Self-Assembled IIINitrides Quantum-Dot Lasers", Recent Patents on Electrical Engineering J, 3, 211217,2010 . 


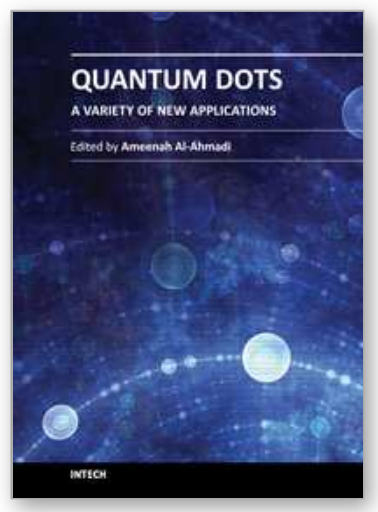

\section{Quantum Dots - A Variety of New Applications}

Edited by Dr. Ameenah Al-Ahmadi

ISBN 978-953-51-0483-4

Hard cover, 280 pages

Publisher InTech

Published online 04, April, 2012

Published in print edition April, 2012

The book "Quantum dots: A variety of a new applications" provides some collections of practical applications of quantum dots. This book is divided into four sections. In section 1 a review of the thermo-optical characterization of CdSe/ZnS core-shell nanocrystal solutions was performed. The Thermal Lens (TL) technique was used, and the thermal self-phase Modulation (TSPM) technique was adopted as the simplest alternative method. Section 2 includes five chapters where novel optical and lasing application are discussed. In section 3 four examples of quantum dot system for different applications in electronics are given. Section 4 provides three examples of using quantum dot system for biological applications. This is a collaborative book sharing and providing fundamental research such as the one conducted in Physics, Chemistry, Biology, Material Science, Medicine with a base text that could serve as a reference in research by presenting up-todate research work on the field of quantum dot systems.

\section{How to reference}

In order to correctly reference this scholarly work, feel free to copy and paste the following:

Hussein B. AL-Husseini (2012). Factors Affecting the Relative Intensity Noise of GaN Quantum Dot Lasers, Quantum Dots - A Variety of New Applications, Dr. Ameenah Al-Ahmadi (Ed.), ISBN: 978-953-51-0483-4, InTech, Available from: http://www.intechopen.com/books/quantum-dots-a-variety-of-new-applications/factorsaffecting-on-the-relative-intensity-noise-from-quantum-dot-laser

\section{INTECH}

open science | open minds

\section{InTech Europe}

University Campus STeP Ri

Slavka Krautzeka 83/A

51000 Rijeka, Croatia

Phone: +385 (51) 770447

Fax: +385 (51) 686166

www.intechopen.com

\section{InTech China}

Unit 405, Office Block, Hotel Equatorial Shanghai

No.65, Yan An Road (West), Shanghai, 200040, China

中国上海市延安西路65号上海国际贵都大饭店办公楼 405 单元

Phone: +86-21-62489820

Fax: $+86-21-62489821$ 
(C) 2012 The Author(s). Licensee IntechOpen. This is an open access article distributed under the terms of the Creative Commons Attribution 3.0 License, which permits unrestricted use, distribution, and reproduction in any medium, provided the original work is properly cited. 